\title{
Homomesy in products of two chains
}

\author{
James Propp 1 "fand Tom Roby ${ }^{2}$ \\ ${ }^{1}$ University of Massachusetts, Lowell, MA, USA \\ ${ }^{2}$ University of Connecticut, Storrs, CT, USA
}

\begin{abstract}
Many cyclic actions $\tau$ on a finite set $\mathcal{S}$ of combinatorial objects, along with a natural statistic $f$ on $\mathcal{S}$, exhibit "homomesy": the average of $f$ over each $\tau$-orbit in $\mathcal{S}$ is the same as the average of $f$ over the whole set $\mathcal{S}$. This phenomenon was first noticed by Panyushev in 2007 in the context of antichains in root posets; Armstrong, Stump, and Thomas proved Panyushev's conjecture in 2011. We describe a theoretical framework for results of this kind and discuss old and new results for the actions of promotion and rowmotion on the poset that is the product of two chains.

Résumé. Plusieurs actions cycliques $\tau$ sur un ensemble fini $\mathcal{S}$ d'objets combinatoires muni d'une statistique naturelle $f$ sur $\mathcal{S}$ démontrent une "homomesie": la moyenne de $f$ sur une orbite de $\tau$ en $\mathcal{S}$ est la même que la moyenne de $f$ sur la totalité de l'ensemble $\mathcal{S}$. Ce phénomène a été d'abord remarqué par Panyushev en 2007 dans le contexte des anti-chaînes dans des posets de racine; Armstrong, Stump, et Thomas ont demontré la conjecture de Panyushev en 2011. On décrit un contexte de travail pour énoncer des résultats de ce genre et on discute de nouveaux et d'anciens résultats pour des actions de "promotion" et "rowmotion" sur le poset qui est le produit de deux chaînes.
\end{abstract}

Keywords: antichains, combinatorial ergodicity, homomesy, orbit, order ideals, poset, product of chains, promotion, rowmotion, sandpile, toggle group.

\section{Introduction}

We begin with the definition of our main unifying concept, and supporting nomenclature.

Definition 1. Given a finite set $\mathcal{S}$ of combinatorial objects, an invertible map $\tau$ from $\mathcal{S}$ to itself, and a function (or "statistic") $f: \mathcal{S} \rightarrow K$ taking values in some field $K$ of characteristic zero, we say the triple $(\mathcal{S}, \tau, f)$ exhibits homomesy iff there exists a constant $c \in K$ such that for every $\tau$-orbit $\mathcal{O} \subset \mathcal{S}$

$$
\frac{1}{\# \mathcal{O}} \sum_{x \in \mathcal{O}} f(x)=c .
$$

In this situation we say that the function $f: \mathcal{S} \rightarrow K$ is homomesic (Greek for "same middle") relative to the action of $\tau$ on $\mathcal{S}$. If $c$ is 0 , we say that $f$ is $\mathbf{0}$-mesic.

\footnotetext{
${ }^{\dagger}$ Partially supported by NSF Grant \#1001905

1365-8050 (c) 2013 Discrete Mathematics and Theoretical Computer Science (DMTCS), Nancy, France
} 
We also apply the term "homomesic" more broadly to situations in which $\mathcal{S}$ is not a set of combinatorial objects or the statistic $f$ takes values in a vector space over a field of characteristic 0 (as in sections 2.2 and 2.3. Homomesy can be restated equivalently as all orbit-averages being equal to the global average:

$$
\frac{1}{\# \mathcal{O}} \sum_{x \in \mathcal{O}} f(x)=\frac{1}{\# \mathcal{S}} \sum_{x \in \mathcal{S}} f(x) .
$$

This is the form in which Panyushev [Pan08] stated his conjecture.

We have found many instances of (2) where the actions $\tau$ and the statistics $f$ are natural ones. Many (but far from all) situations that support examples of homomesy also support examples of the cyclic sieving phenomenon of Reiner, Stanton, and White [RSW04]. Examples of homomesy are given starting with Section 2

At the stated level of generality this notion appears to be new, but specific instances can be found in earlier literature. In particular, in 2007, Panyushev [Pan08] conjectured and in 2011, Amstrong, Stump, and Thomas [AST11] proved that if $\mathcal{S}$ is the set of antichains in the root poset of a finite Weyl group, $\Phi$ is the operation variously called the Brouwer-Schrijver map [BS74], the Fon-der-Flaass map [Fon93, CF95], the reverse map [Pan08], Panyushev complementation [AST11], and rowmotion [SW12], and $f(A)$ is the cardinality of the antichain $A$, then $(\mathcal{S}, \Phi, f)$ satisfies (2).

Our main results for this paper involve studying the action of this rowmotion operator and also the promotion operator on the poset $P=[a] \times[b]$, the product of two chains. (Here and throughout this article we use $[n]$ to denote the set $\{1,2, \ldots, n\}$ and the associated $n$-element poset.) We show that the statistic $f:=\# A$, the size of the antichain, is homomesic with respect to the promotion operator, and that the statistic $f=\# I(A)$, the size of the corresponding order ideal, is homomesic with respect to both the promotion and rowmotion operators.

Although these results are of intrinsic interest, we think the main contribution of the paper is its identification of homomesy as a phenomenon that (as we expect future articles to show) occurs quite widely. Within any linear space of functions on $\mathcal{S}$, the functions that are homomesic under $\tau$, like the functions that are invariant under $\tau$, form a subspace, and there is a loose sense in which the notions of invariance and homomesy (or, more strictly speaking, 0-mesy) are dual; an extremely clean case of this duality is outlined in subsection 2.2. This extended abstract gives a general overview of the broader picture as well as a few specific examples done in more detail for the operators of promotion and rowmotion on the poset $[a] \times[b]$.

The authors are grateful to Drew Armstrong, Anders Björner, Barry Cipra, Karen Edwards, Robert Edwards, Darij Grinberg, Shahrzad Haddadan, Svante Linusson, Vic Reiner, Ralf Schiffler, Richard Stanley, Jessica Striker, Peter Winkler and Ben Young for useful conversations. Mike LaCroix wrote fantastic postscript code to generate animations and pictures that illustrate our maps operating on order ideals on products of chains. Ben Young also provided a diagram which we modified for Figure 6 , Two anonymous referees made very helpful suggestions for improving this abstract. Several of our ideas were first incubated at meetings of the long-running Cambridge Combinatorial Coffee Club (CCCC), organized by Richard Stanley.

\section{Examples of Homomesy}

\subsection{Rotation of bit-strings}

We begin with a simple concrete example to clarify the definition. 


$$
\begin{aligned}
& \ldots \quad \stackrel{\tau}{\rightarrow} 0011 \stackrel{\tau}{\rightarrow} 0110 \stackrel{\tau}{\rightarrow} \quad 1100 \stackrel{\tau}{\rightarrow} \quad 1001 \quad \stackrel{\tau}{\rightarrow} \quad \ldots \\
& \begin{array}{rrrr}
f \downarrow & f \downarrow & f \downarrow & f \downarrow \\
& 2 & 4 &
\end{array} \\
& \ldots \quad \stackrel{\tau}{\rightarrow} 1010 \quad \stackrel{\tau}{\rightarrow} 0101 \stackrel{\tau}{\rightarrow} \quad \ldots \\
& f \downarrow \quad f \downarrow \\
& 3 \quad 1
\end{aligned}
$$

Fig. 1: The two orbits of the action of the cyclic shift $\tau=C_{L}$ on binary strings consisting of two 0's and two 1's. The average value of the inversion statistic $f$ is $(0+2+4+2) / 4=2$ on the orbit of size 4 and $(3+1) / 2=2$ on the orbit of size 2 .

Example 1. Let $\mathcal{S}:=\left(\begin{array}{c}{[n]} \\ k\end{array}\right)$, thought of as the set of length $n$ binary strings with $k$ 1's and $n-k 0$ 's. Set $f(b):=\operatorname{inv}(b):=\#\left\{i<j: b_{i}>b_{j}\right\}$ and $\tau:=C_{L}: \mathcal{S} \rightarrow \mathcal{S}$ the leftward cyclic shift operator given by $b=b_{1} b_{2} \cdots b_{n} \mapsto b_{2} b_{3} \cdots b_{n} b_{1}$. Then over any orbit $\mathcal{O}$ we have

$$
\frac{1}{\# \mathcal{O}} \sum_{s \in \mathcal{O}} f(s)=\frac{k(n-k)}{2}=\frac{1}{\# \mathcal{S}} \sum_{s \in \mathcal{S}} f(s) .
$$

This fact can be proved in isolation, but it also follows from one of our results from Section 3 , with $a=k$ and $b=n-k$. More specifically, we have a bijection between order-ideals in the poset $P=[a] \times[b]$ and strings consisting of $a-1$ 's and $b+1$ 's (which in turn correspond to bit strings, if one replaces the -1 's by 0 's). Then promotion on $J(P)$ is equivariant with leftward cyclic shift on strings, and the cardinality of an order ideal is equal to the number of inversions in the associated string. Theorem 11 then yields the claimed result on bit-strings.

In the specfic case $n=4, k=2$, the six-element set decomposes into two orbits, shown in Figure 1 As frequently happens, not all orbits are the same size; however, one could also view the orbit of size 2 as a "multiset orbit" of size 4, cycling through the same set of elements twice. This perspective, where we view all orbits as multiset orbits of the same size, facilitates the discussion of certain comparisons.

\subsection{Linear actions on vector spaces}

Let $V$ be a (not necessarily finite-dimensional) vector space over a field $K$ of characteristic zero, and define $f(v)=v$ (that is, our "statistic" is just the identity function). Let $T: V \rightarrow V$ be a linear map such that $T^{n}=I$ (the identity map on $V$ ) for some fixed $n \geq 1$ (i.e., $I-T^{n}$ is the 0 -map). Say $v$ is invariant under $T$ if $T v=v$ (i.e., $v$ is in the kernel of $I-T)$ and 0 -mesic under $T$ if $\left(v+T v+\cdots+T^{n-1} v\right) / n=0$ (i.e., $v$ is in the kernel of $I+T+T^{2}+\cdots+T^{n-1}$ ). Every $v \in V$ can be written uniquely as the sum of an invariant vector $\bar{v}$ and a 0 -mesic vector $\hat{v}$ (specifically, one can check that $\bar{v}=\left(v+T v+\cdots+T^{n-1} v\right) / n$ and $\hat{v}=v-\bar{v}$ work, and no other solution is possible because that would yield a nonzero vector that is both invariant and 0-mesic, which does not exist). In representation-theoretic terms, we are applying symmetrization to $v$ to extract from it the invariant component $\bar{v}$ associated with the trivial representation of the cyclic group, and the homomesic (0-mesic) component $\hat{v}$ consists of everything else.

This picture relates more directly to our earlier definition if we use the dual space $V^{*}$ of linear functionals on $V$ as the set of statistics on $V$. As a concrete example, let $V=\mathbf{R}^{n}$ and let $T$ be the cyclic 
shift of coordinates sending $\left(x_{1}, x_{2}, \ldots, x_{n}\right)$ to $\left(x_{n}, x_{1}, \ldots, x_{n-1}\right)$. The $T$-invariant functionals form a 1dimensional subspace of $V^{*}$ spanned by the functional $\left(x_{1}, x_{2}, \ldots, x_{n}\right) \mapsto x_{1}+x_{2}+\ldots+x_{n}$, while the homomesic functionals form an $(n-1)$-dimensional subspace of $V^{*}$ spanned by the $n-1$ functionals $\left(x_{1}, x_{2}, \ldots, x_{n}\right) \mapsto x_{i}-x_{i+1}($ for $1 \leq i \leq n-1)$.

\subsection{Sandpile dynamics}

Let $G$ be a directed graph with vertex set $V$. For $v \in V$ let outdeg $(v)$ be the number of directed edges emanating from $v$, and for $v, w \in V$ let $\operatorname{deg}(v, w)$ be the number of directed edges from $v$ to $w$ (which we will permit to be larger than 1, even when $v=w$ ). Define the combinatorial Laplacian of $G$ as the matrix $\Delta$ (with rows and columns indexed by the vertices of $V$ ) whose $v, v$ th entry is outdeg $(v)-\operatorname{deg}(v, v)$ and whose $v, w$ th entry for $v \neq w$ is $-\operatorname{deg}(v, w)$. Specify a global sink $t$ with the property that for all $v \in V$ there is a forward path from $v$ to $t$, let $V^{-}=V \backslash\{t\}$, and let $\Delta^{\prime}$ (the reduced Laplacian) be the matrix $\Delta$ with the row and column associated with $t$ removed. By the Matrix-Tree theorem, $\Delta^{\prime}$ is nonsingular. A sandpile configuration on $G$ (with sink at $t$ ) is a function $\sigma$ from $V^{-}$to the nonnegative integers. (For more background on sandpiles, see Holroyd, Levine, Mészáros, Peres, Propp, \& Wilson [HLMPPW08].) We say $\sigma$ is stable if $\sigma(v)<\operatorname{outdeg}(v)$ for all $v \in V^{-}$. For any sandpile-configuration $\sigma$, Dhar's least-action principle for sandpile dynamics (see Levine \& Propp [LP10]) tells us that the set of nonnegative-integervalued functions $u$ on $V^{-}$such that $\sigma-\Delta^{\prime} u$ is stable has a minimal element $\phi=\phi(\sigma)$ in the natural (pointwise) ordering; we call $\phi$ the firing vector for $\sigma$ and we call $\sigma-\Delta^{\prime} \phi$ the stabilization of $\sigma$, denoted by $\sigma^{\circ}$. If we choose a source vertex $s \in V^{-}$, then we can define an action on sandpile configurations via $\tau(\sigma)=\left(\sigma+1_{s}\right)^{\circ}$, where $1_{v}$ denotes the function that takes the value 1 at $v$ and 0 elsewhere. Say that $\sigma$ is recurrent (relative to $s$ ) if $\tau^{m}(\sigma)=\sigma$ for some $m>0$. (This notion of recurrence is slightly weaker than that of [HLMPPW08]; they are equivalent when every vertex is reachable by a path from s.) Then $\tau$ restricts to an invertible map from the set of recurrent sandpile configurations to itself. Let $f(\sigma)=\phi\left(\sigma+1_{s}\right)$. Since $\tau(\sigma)=\sigma+1_{s}-\Delta^{\prime} f(\sigma)$ we have $\tau(\sigma)-\sigma=1_{s}-\Delta^{\prime} f(\sigma)$; if we average this relation over all $\sigma$ in a particular $\tau$-orbit, the left side telescopes, giving $0=1_{s}-\Delta^{\prime} \bar{f}$, where $\bar{f}$ denotes the average of $f$ over the orbit. Hence:

Proposition 2. For the action of $\tau$ on recurrent sandpile configurations, the function $f: \sigma \mapsto \phi\left(\sigma+1_{s}\right)$ is homomesic, and its orbit-average is the function $f^{*}$ on $V^{-}$such that $\Delta^{\prime} f^{*}=1_{s}$ (unique because $\Delta^{\prime}$ is nonsingular).

Example 2. Figure 2 shows an example of the $\tau$-orbits for the case where $G$ is the bidirected cycle graph with vertices $1,2,3$, and 4 , with a directed edge from $i$ to $j$ iff $i-j= \pm 1 \bmod 4$; here the discrete Laplacian is

$$
\Delta=\left(\begin{array}{rrrr}
2 & -1 & 0 & -1 \\
-1 & 2 & -1 & 0 \\
0 & -1 & 2 & -1 \\
-1 & 0 & -1 & 2
\end{array}\right)
$$

Let the source be $s=2$ and global sink be $t=4$. The sandpile configuration $\sigma$ is represented by the triple $(\sigma(1), \sigma(2), \sigma(3))$. The four recurrent configurations $\sigma$ are $(1,0,1),(1,1,1),(0,1,1)$, and $(1,1,0)$, and the respective firing vectors $f(\sigma)$ are $(0,0,0),(1,2,1),(0,1,1)$, and $(1,1,0)$. The average value of the firing vector statistic $f$ is $f^{*}=\left(\frac{1}{2}, 1, \frac{1}{2}\right)$ on each orbit. Treating $f^{*}$ as a column vector and multiplying 


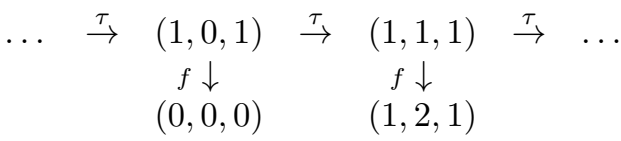

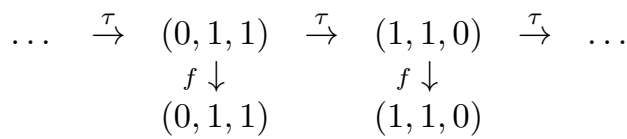

Fig. 2: The two orbits in the action of the sandpile map $\tau$ on recurrent configurations on the cycle graph of size 4, with source at 2 and sink at 4 . There are two orbits, each of size 2 , and the average of $f$ along each orbit is $(1 / 2,1,1 / 2)$.

on the left by $\Delta^{\prime}$ gives the column vector $(0,1,0)=1_{s}$ :

$$
\left(\begin{array}{rrr}
2 & -1 & 0 \\
-1 & 2 & -1 \\
0 & -1 & 2
\end{array}\right)\left(\begin{array}{c}
1 / 2 \\
1 \\
1 / 2
\end{array}\right)=\left(\begin{array}{l}
0 \\
1 \\
0
\end{array}\right) \text {. }
$$

Similar instances of homomesy were known for a variant of sandpile dynamics called rotor-router dynamics; see Holroyd-Propp [HP10]. It was such instances of homomesy that led the second author to seek instances of the phenomenon in other, better-studied areas of combinatorics.

\section{Promotion and rowmotion in products of two chains}

For a finite poset $P$, we let $J(P)$ denote the set of order ideals (or down-sets) of $P, F(P)$ denote the set of (order) filters (or up-sets) of $P$, and $\mathcal{A}(P)$ be the set of antichains of $P$. (For standard definitions and notation about posets and ideals, see Stanley [EC1].) There is a bijection $J(P) \leftrightarrow \mathcal{A}(P)$ given by taking the maximal elements of $I \in J(P)$ or conversely by taking the order ideal geneated by an antichain $A \in \mathcal{A}(P)$. Similarly, there is a bijection $F(P) \leftrightarrow \mathcal{A}(P)$. Composing these with the complementation bijection between $J(P)$ and $F(P)$ leads to an interesting map that has been studied in several contexts [BS74, Fon93, CF95, Pan08, AST11, SW12], namely $\Phi_{A}:=\mathcal{A}(P) \rightarrow J(P) \rightarrow F(P) \rightarrow \mathcal{A}(P)$ and the companion map $\Phi_{J}:=J(P) \rightarrow F(P) \rightarrow \mathcal{A}(P) \rightarrow J(P)$, where the subscript indicates whether we consider the map to be operating on antichains or order ideals. We often drop the subscript and just write $\Phi$ when context makes clear which is meant. Following Striker and Williams [SW12] we call this map rowmotion.

Let $[a] \times[b]$ denote the poset that is a product of chains of lengths $a$ and $b$. Figure 5 shows an orbit of the action of $\Phi_{J}$ starting from the ideal generated by the antichain $\{(2,1)\}$. Note that the elements of $[4] \times[2]$ here are represented by the squares rather than the points in the picture, with covering relations represented by shared edges. One can also view this as an orbit of $\Phi_{A}$ if one just considers the maximal elements in each shaded order ideal.

This section contains our main specific results, namely that the following triples exhibit homomesy:

$$
\left(J([a] \times[b]), \Phi_{J}, \# I\right) ; \quad\left(\mathcal{A}([a] \times[b]), \Phi_{A}, \# A\right) ; \text { and } \quad\left(J([a] \times[b]), \partial_{J}, \# I\right) .
$$

Here $\partial_{J}$ is the promotion operator to be defined in the next subsection, and \#I (resp. \#A) denotes the 
statistic on $J(P)$ (resp. $\mathcal{A}(P)$ ) that is the cardinality of the order ideal $I$ (resp. the antichain $A$ ). All maps operate on the left (e.g., we write $\partial_{J} I$, not $I \partial_{J}$ ).

\subsection{Background on the toggle group}

Several of our examples arise from the toggle group of a finite poset (first explicitly defined in [SW12]; see also [CF95, Sta09, SW12]). We review some basic facts and provide some pointers to relevant literature.

Definition 3. Given $x \in P$, we define the toggle operation $\sigma_{x}: J(P) \rightarrow J(P)$ ("toggling at $x$ ") via

$$
\sigma_{x}(I)= \begin{cases}I \triangle\{x\} & \text { if } I \triangle\{x\} \in J(P) \\ I & \text { otherwise. }\end{cases}
$$

Proposition 4 ([CF95]). (a) For every $x \in P, \sigma_{x}$ is an involution, i.e., $\sigma_{x}^{2}=1$.

(b) For every $x, y \in P$ where neither $x$ covers $y$ nor $y$ covers $x$, the toggles commute, i.e., $\sigma_{x} \sigma_{y}=\sigma_{y} \sigma_{x}$.

Proposition 5 ([CF95]). Let $x_{1}, x_{2}, \ldots, x_{n}$ be any linear extension (i.e., any order-preserving listing of the elements) of a poset $P$ with $n$ elements. Then the composite map $\sigma_{x_{1}} \sigma_{x_{2}} \cdots \sigma_{x_{n}}$ coincides with the rowmotion operator $\Phi_{J}$.

Corollary 6 ([SW12], Cor. 4.9). Let $P$ be a graded poset of rank $r$, and set $T_{k}:=\prod_{|x|=k} \sigma_{x}$, the product of all the toggles of elements of fixed rank $k$. (This is well-defined by Proposition 4) Then the composition $T_{1} T_{2} \cdots T_{r}$ coincides with $\Phi_{J}$, i.e., rowmotion is the same as toggling by ranks from top to bottom.

We focus on the case $P=[a] \times[b]$, whose elements we write as $(k, \ell)$; the Hasse diagram of $P$ consists of the points $(i, j):=(\ell-k, \ell+k-2)(1 \leq k \leq a, 1 \leq \ell \leq b)$ so that the poset-elements $(k, \ell)=(1,1)$, $(a, 1),(1, b)$, and $(a, b)$ are respectively the bottom, left, right, and top elements of the Hasse diagram.

Definition 7. In this situation, we call the sets with constant $j$ ranks (in accordance with standard poset terminology), sets with constant $i$ files, sets with constant $j-i$ positive fibers, and sets with constant $j+i$ negative fibers. (The words "positive" and "negative" indicate the slopes of the lines on which the fibers lie in the Hasse diagram.) More specifically, the element $(k, \ell) \in[a] \times[b]$ belongs to rank $k+\ell-2$, to file $\ell-k$, to positive fiber $k$, and to negative fiber $\ell$.

To each order ideal $I \in J([a] \times[b])$ we associate a lattice path of length $a+b$ joining the points $(-a, a)$ and $(b, b)$ in the plane, where each step is of type $(i, j) \rightarrow(i+1, j+1)$ or of type $(i, j) \rightarrow(i+1, j-1)$, as follows. Given $1 \leq k \leq a$ and $1 \leq \ell \leq b$, represent $(k, \ell) \in[a] \times[b]$ by the square centered at $(\ell-k, \ell+k-1)$ with vertices $(\ell-k, \ell+k-2),(\ell-k, \ell+k),(\ell-k-1, \ell+k-1)$, and $(\ell-k+1, \ell+k-1)$. Then the squares representing the elements of the order ideal $I$ form a "Russianstyle" Young diagram whose upper border is a path joining some point on the line of slope -1 to some point on the line of slope +1 . Adding extra edges of slope -1 at the left and extra edges of slope +1 at the right, we get a path joining $(-a, a)$ to $(b, b)$. See Figure 5 for several examples of this correspondence.

Definition 8. We can think of this path as the graph of a (real) piecewise-linear function $h_{I}:[-a, b] \rightarrow$ $[0, a+b]$; we call this function (or its restriction to $[-a, b] \cap \mathbf{Z}$ ) the height function representation of the ideal I. To this height function we can in turn associate a word consisting of $a-1$ 's and $b+1$ 's, whose ith term (for $1 \leq i \leq a+b)$ is $h_{I}(i-a)-h_{I}(i-a-1)= \pm 1$; we call this the sign-word associated with the order ideal $I$.

Note that the sign-word simply lists the slopes of the segments making up the path, and that either the sign-word or the height-function encodes all the information required to determine the order ideal. 
Proposition 9. Let $I \in J([a] \times[b])$ correspond with height function $h_{I}:[-a, b] \rightarrow \mathbf{R}$. Then

$$
\sum_{k=-a}^{b} h_{I}(k)=\frac{a(a+1)}{2}+\frac{b(b+1)}{2}+2 \# I .
$$

So to prove that the cardinality of $I$ is homomesic, it suffices to prove that the function $h_{I}(-a)+$ $h_{I}(-a+1)+\cdots+h_{I}(b)$ is homomesic (where our combinatorial dynamical system acts on height functions $h$ via its action on order ideals $I$ ).

\subsection{Promotion in products of two chains}

In general a ranked poset $P$ may not have an embedding in $\mathbf{Z} \times \mathbf{Z}$ that allows files to be defined; when they are, however, then all toggles corresponding to elements within the same file commute by Proposition 4 . so their product is a well defined operation on $J(P)$. This allows one to define an operation on $J(P)$ by successively toggling all the files from left to right, in analogy to Corollary 6.

Theorem 10 (Striker-Williams [SW12, $\S 6.1]$ ). Let $x_{1}, x_{2}, \ldots, x_{n}$ be any enumeration of the elements $(k, \ell)$ of the poset $[a] \times[b]$ arranged in order of increasing $\ell-k$. Then the action on $J(P)$ given by $\partial:=\sigma_{x_{n}} \circ \sigma_{x_{n-1}} \circ \cdots \circ \sigma_{x_{1}}$ viewed as acting on the paths (or binary strings representing them) is just $a$ leftward cyclic shift.

Striker and Williams call this well-defined composition $\partial$ promotion (since it is related to Schützenberger's notion of promotion on linear extensions of posets). They show that it is conjugate to rowmotion in the toggle group, obtaining a much simpler bijection to prove Panyushev's conjecture in Type A, and generalizing an equivariant bijection for $[a] \times[b]$ of Stanley [Sta09, remark after Thm 2.5]. This definition and their results apply more generally to the class they define of $r c$-posets, whose elements fit neatly into "rows" and "columns" (which we call here "ranks" and "files"). As with $\Phi$, we can think of $\partial$ as operating either on $J(P)$ or $\mathcal{A}(P)$, adding subscripts $\partial_{J}$ or $\partial_{A}$ if necessary. Since the cyclic left-shift has period $a+b$, so does $\partial$.

Theorem 11. The cardinality of order ideals is homomesic under the action of promotion $\partial_{J}$.

Proof: To show that \#I is homomesic, by Proposition 9 it suffices to show that $h_{I}(k)$ is homomesic for all $-a \leq k \leq b$. Note that here we are thinking of $I$ as varying over $J(P)$, and $h_{I}$ as being a function-valued function on $J(P)$.

We can write $h_{I}(k)$ as the telescoping sum $h_{I}(-a)+\left(h_{I}(-a+1)-h_{I}(-a)\right)+\left(h_{I}(-a+2)-h_{I}(-a+\right.$ 1)) $+\cdots+\left(h_{I}(k)-h_{I}(k-1)\right)$; to show that $h_{I}(k)$ is homomesic for all $k$, it will be enough to show that all the increments $h_{I}(k)-h_{I}(k-1)$ are homomesic. Note that these increments are precisely the terms of the sign-word of $I$. Create a square array with $a+b$ rows and $a+b$ columns, where the rows are the sign-words of $I$ and its successive images under the action of $\partial$; each row is just the cyclic left-shift of the row before. Since each row contains $a-1$ 's and $b+1$ 's, the same is true of each column. Thus, for all $k$, the average value of the $k$ th terms of the sign-words of $I, \partial I, \partial^{2} I, \ldots, \partial^{a+b-1} I$ is $(b-a) /(b+a)$. This show that the increments are homomesic, as required, which suffices to prove the theorem.

Our proof actually shows the more refined result that the restricted cardinality functions $\#(I \cap S)$ where $S$ is any file of $[a] \times[b]$ are homomesic with respect to the action of $\partial_{J}$.

The next example shows that the cardinality of the antichain $A_{I}$ associated with the order ideal $I$ is not homomesic under the action of promotion $\partial$. 

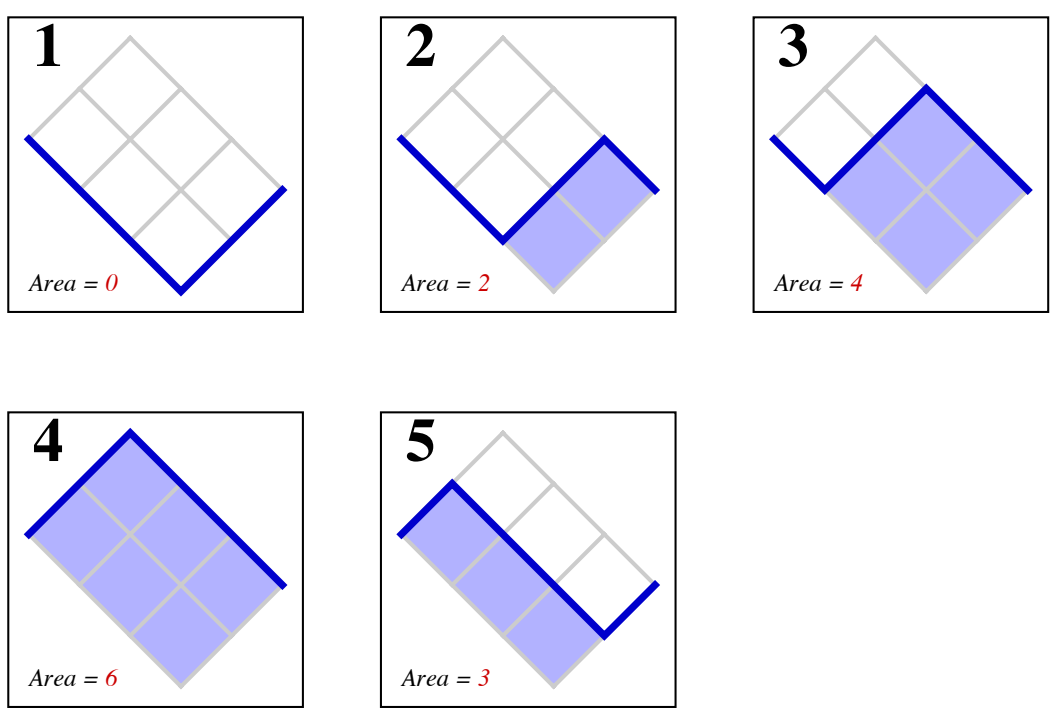

\section{$(0+2+4+6+3) / 5=3$}

Fig. 3: One promotion orbit in $J([3] \times[2])$

Example 3. Consider the two promotion orbits of $\partial_{A}$ shown in Figures 3 and 4 Although the statistic \#I is homomesic, giving an average of 3 in both cases, the statistic $\# A$ averages to $\frac{1}{5}(0+1+1+1+1)=$ $\frac{4}{5}$ in the first orbit and to $\frac{1}{5}(1+2+2+1+2)=\frac{8}{5}$ in the second.

\subsection{Rowmotion in products of two chains}

Unlike promotion, the rowmotion operator turns out to exhibit homomesy with respect to both the statistic that counts the size of an order ideal and the statistic that counts the size of an antichain.

\subsubsection{Rowmotion on order ideals in $J([a] \times[b])$}

We can describe rowmotion nicely in terms of the sign-word. We define blocks within the sign-word as occurrences of the subword $-1,+1$ (that is, $a-1$ followed immediately by $a+1$ ). Once we have found all the blocks, we identify all the gaps between the blocks, where a gap is bounded by two consecutive blocks, or between the beginning of the word and the first block, or between the last block and the end of the word. (In the case where there are no blocks at all, the entire word is considered a gap.) To apply rowmotion to a sign-word, reverse all the blocks and all the gaps. For example, consider the binary word $-1,+1,+1,-1,-1,-1,+1,+1$. To apply rowmotion to it, we first divide it into blocks and gaps as $-1,+1,|+1,-1,-1|-1,+1,, \mid+1$, and then reverse each block and gap in place, obtaining $+1,-1, \mid$ $-1,-1,+1,|+1,-1|+$,1 , or (dropping the dividers) $+1,-1,-1,-1,+1,+1,-1,+1$. Note that the 

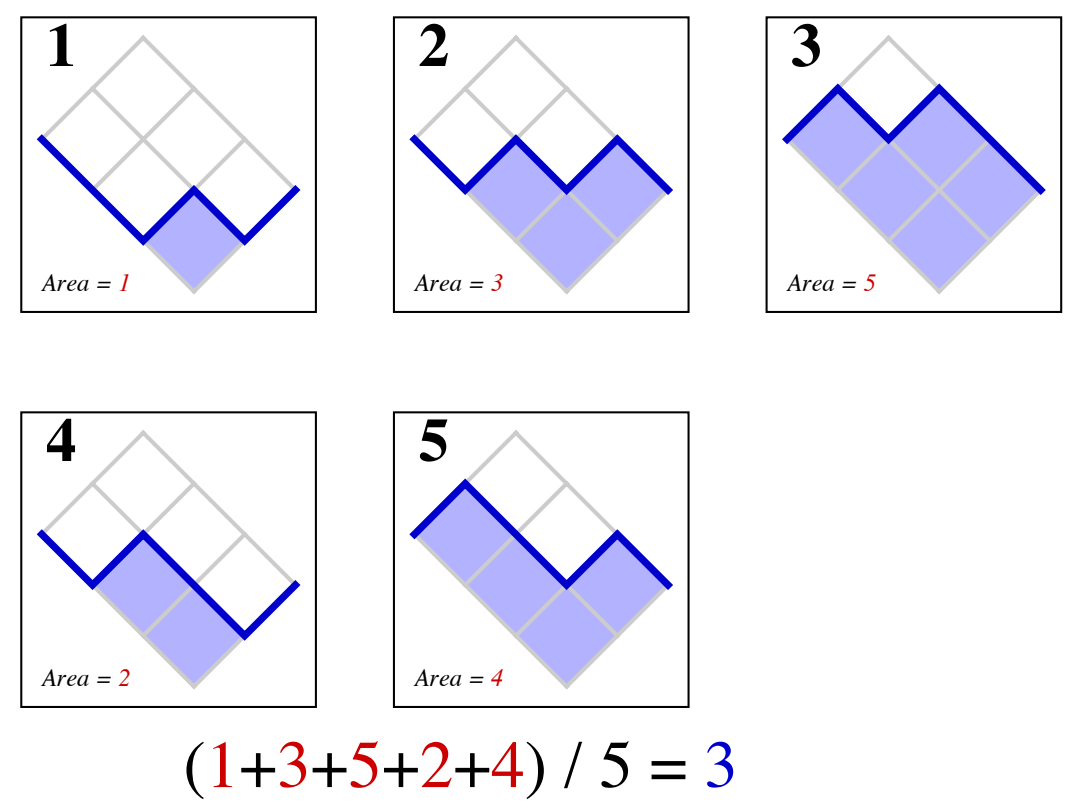

Fig. 4: The other promotion orbit in $J([3] \times[2])$

dividers correspond to the red dots in Figure 5, so one can visualize $\Phi_{J}$ as reversing ( $180^{\circ}$ rotation of) each lattice-path segment that corresponds to a block or a gap in the sign-word. (See animations within talk slides at http: //www. math.uconn.edu/ troby/combErg2012kizugawa.pdf])

It turns out that all we really need to know for purposes of proving homomesy is that the sign-word for $I$ has $-1,+1$ in a pair of adjacent positions if and only if the sign-word for $\Phi I$ has $+1,-1$ in the same two positions. This can be seen directly for $J([a] \times[b])$ from the description of $\Phi_{J}$ given at the start of Section 3. (See also Figure 5) This situation occurs if and only if the antichain $A(\Phi(I))$ contains an element in the associated file of $[a] \times[b]$.

Theorem 12. The cardinality of order ideals is homomesic under the action of rowmotion $\Phi_{J}$.

Proof: As in the previous section, to prove that $\# I$ is homomesic under rowmotion, it suffices to prove that all the increments $h_{I}(k)-h_{I}(k-1)$ are homomesic. A result of Fon-der-Flaass [Fon93, Theorem 2], states that the size of any $\Phi$-orbit in $[a] \times[b]$ is a divisor of $a+b$, so this is equivalent to showing that for all $k$, the $k$ th element of the sign-word of $\sum_{m=0}^{a+b-1} \Phi^{m} I$ under the action of rowmotion is independent of $I$.

Create a square array with $a+b$ rows and $a+b$ columns, where the rows are the sign-words of $I$ and its successive images under the action of $\Phi$. Consider any two consecutive columns of the array, and the width-2 subarray they form. There are just four possible combinations of values in a row of the subarray: 

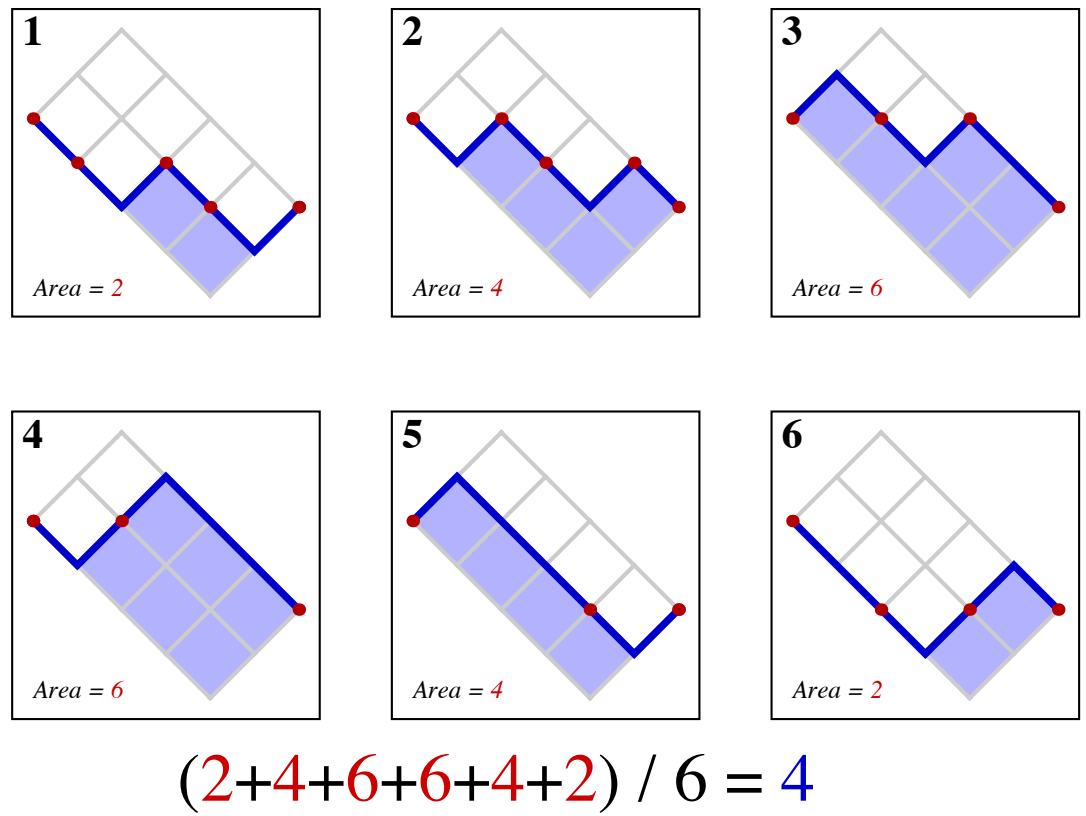

Fig. 5: A rowmotion orbit in $J([4] \times[2])$

$(+1,+1),(+1,-1),(-1,+1)$, and $(-1,-1)$. However, we have just remarked that a row is of type $(-1,+1)$ if and only if the next row is of type $(+1,-1)$ (where we consider the row after the bottom row to be the top row). Hence the number of rows of type $(-1,+1)$ equals the number of rows of type $(+1,-1)$. It follows that any two consecutive column-sums of the full array are equal, since other row types contribute the same value to each column sum. That is, within the original square array, every two consecutive columns have the same column-sum. Hence all columns have the same column-sum. This common value of the column-sum must be $1 /(a+b)$ times the grand total of the values of the square array. But since each row contains $a-1$ 's and $b+1$ 's, each row-sum is $b-a$, so the grand total is $(a+b)(b-a)$, and each column-sum is $b-a$. Since this is independent of which rowmotion orbit we are in, we have proved homomesy for elements of the sign-word of $I$ as $I$ varies over $J([a] \times[b])$, and this gives us the desired result about $\# I$, just as in the proof of Theorem 11.

\subsubsection{Rowmotion on antichains in $\mathcal{A}([a] \times[b])$}

In his survey article on promotion and evacuation, Stanley [Sta09, remark after Thm 2.5] gave a concrete equivariant bijection between rowmotion $\Phi_{A}$ acting on antichains in $\mathcal{A}([a] \times[b])$ and cyclic rotation of certain binary strings. Armstrong (private communication) gave a variant description that clarified the correspondence, which he learned from Thomas and which we use in what follows. 


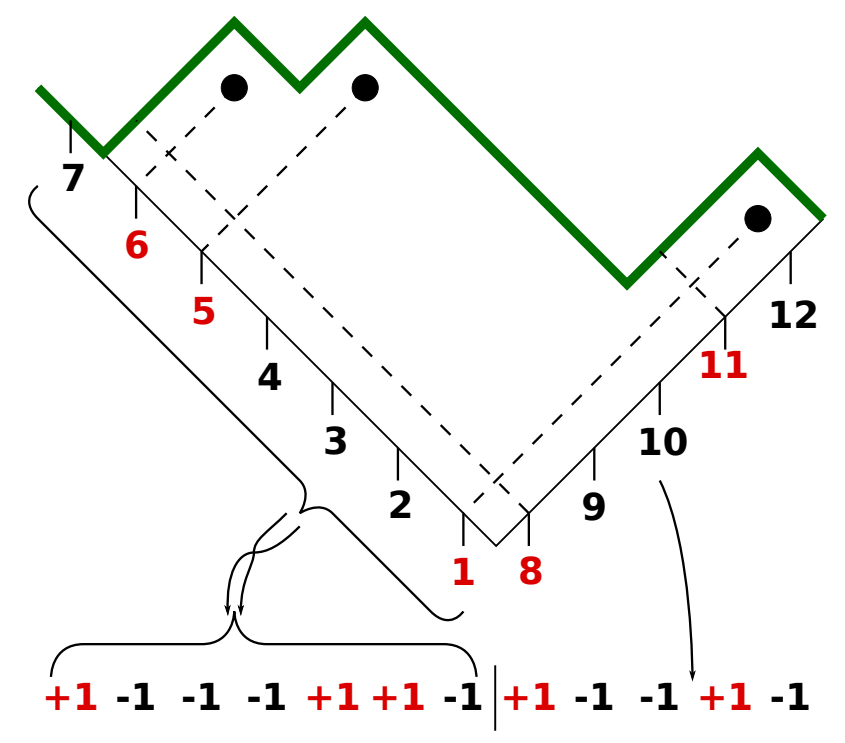

Fig. 6: The Stanley-Thomas word for a 3-element antichain in $\mathcal{A}([7] \times[5])$.

Definition 13. Fix $a, b$, and $n=a+b$. Call sets in $[a] \times[b]$ of the form $\{(k, \ell): \ell \in[b]\}$ (with $k$ fixed) rows and sets of the form $\{(k, \ell): k \in[a]\}$ (with $\ell$ fixed) columns. Define the Stanley-Thomas word $w(A)$ of an antichain $A$ in $[a] \times[b]$ to be $w_{1} w_{2} \cdots w_{a+b} \in\{-1,+1\}^{a+b}$ with

$w_{i}:= \begin{cases}+1, & \text { if } A \text { has an element in row } i(i \in[a]) \text { or A has NO element in column } i(a+1 \leq i \leq n) ; \\ -1 & \text { otherwise. }\end{cases}$

Example 4. As illustrated in Figure 6 let $A=\{(1,5),(5,3),(6,2)\}$. By definition, the StanleyThomas word $w(A)$ should have +1 in entries 1,5 , and 6 (rows where $A$ appears) and in entries 8 and 11 (columns where $A$ does not appear, with indices shifted by $7=a$ ). Indeed one sees that $w(A)=+1,-1,-1,-1,+1,+1,-1, \mid+1,-1,-1,+1,-1$ (where the divider between $a$ and $a+1$ is just for ease of reading). Note that applying rowmotion gives $A^{\prime}=\Phi(A)=\{(2,4),(6,3),(7,1)\}$ with Stanley-Thomas word $w\left(A^{\prime}\right)=-1,+1,-1,-1,-1,+1,+1, \mid-1,+1,-1,-1,+1=C_{R} w(A)$, the rightward cyclic shift of $w(A)$.

Proposition 14 (Stanley-Thomas). The correspondence $A \longleftrightarrow w_{A}$ is a bijection from $\mathcal{A}([a] \times[b])$ to binary words $w \in\{-1,+1\}^{a+b}$ with exactly $a-1$ 's and $b+1$ 's. Furthermore, this bijection is equivariant with respect to the actions of rowmotion $\Phi_{A}$ and rightward cyclic shift $C_{R}$.

Note that the classical result that $\Phi_{A}^{a+b}$ is the identity map follows immediately.

Theorem 15. The cardinality of antichains is homomesic under the action of rowmotion $\Phi_{A}$.

Proof: It suffices to prove a more refined claim, namely, that if $S$ is any row or column of $[a] \times[b]$, the cardinality of $A \cap S$ is homomesic under the action of rowmotion on $A$. By the previous result, rowmotion 
corresponds to cyclic shift of the Stanley-Thomas word, and the entries in the Stanley-Thomas word tell us which fibers (rows or columns) contain an element of $A$ and which do not. Specifically, for $1 \leq k \leq a$, if $S$ is the $k$ th row, then $A$ intersects $S$ iff the $k$ th symbol of the Stanley-Thomas word is a +1 . Since the Stanley-Thomas word contains $a-1$ 's and $b+1$ 's, the multiset orbit of $A$ of size $a+b$ has exactly $b$ elements that are antichains that intersect $S$. That is, the sum of $\#(A \cap S)$ over the multiset orbit of size $a+b$ is exactly $b$, for each of the $a$ rows of $[a] \times[b]$. Summing over all the rows, we see that the sum of

$\# A$ over the multiset orbit is $a b$. Hence \#A is homomesic with average $a b /(a+b)$.

\section{References}

[Arm06] D. Armstrong, Generalized noncrossing partitions and combinatorics of Coxeter groups, Mem. Amer. Math. Soc. 202 (2006), no. 949.

[AST11] D. Armstrong, C. Stump and H. Thomas, A uniform bijection between nonnesting and noncrossing partitions, preprint, available at arXiv:math/1101.1277v2 (2011).

[BS74] A. Brouwer and A. Schrijver, On the period of an operator, defined on antichains, Math. Centrum report ZW 24/74 (1974).

[CF95] P. Cameron and D.G. Fon-Der-Flaass, Orbits of Antichains Revisited, Europ. J. Comb. 16 (1995), $545-554$.

[Fon93] D.G. Fon-Der-Flaass, Orbits of Antichains in Ranked Posets, Europ. J. Comb. 14 (1993), 17-22.

[HP10] A. Holroyd and J. Propp, Rotor walks and Markov chains, in Algorithmic Probability and Combinatorics, American Mathematical Society (2010), 105-126.

[HLMPPW08] A. Holroyd, L. Levine, K. Mészáros, Y. Peres, J. Propp, and D. Wilson, Chip-Firing and Rotor-Routing on Directed Graphs, Progress in Probability, 60 (2008), 331-364.

[LP10] L. Levine and J. Propp, What is a... sandpile?, Notices of the AMS 57(8) (2010), 976-979.

[Pan08] D.I. Panyushev, On orbits of antichains of positive roots, Europ. J. Combin. 30 (2009), no. 2, $586-594$.

[RSW04] V. Reiner, D. Stanton, and D. White, The cyclic sieving phenomenon, J. Combin. Theory Ser. A 108 (2004), 17-50.

[EC1] R. Stanley, Enumerative Combinatorics Volume 1, 2nd Ed., no. 49 in Cambridge Studies in Advanced Mathematics, Cambridge University Press, 2011.

[Sta09] R. Stanley, Promotion and Evacuation, Electronic J. Comb. 16(2) (2009), \#R9.

[SW12] J. Striker and N. Williams, Promotion and rowmotion, Europ. J. Comb. 33 (2012), 1919-1942. 